\title{
First case of multiple primary cardiac leiomyosarcoma on a 17 years old boy, evaluated by cardiac MR imaging
}

\begin{abstract}
In this case report we present the history of a patient with Cardiac mass detected at echocardiography. By further evaluation, the patient has undergone cardiac magnetic resonance (MR) imaging prior to cardiac surgery. This study can therefore contribute to the diagnosis of multiple cardiac masses and its relationship to other cardiac structures. These assessments are important to plan strategy of surgical intervention. MR Imaging technique offers the ability to enable discrimination of different tissue characteristics. The use of contrast material MR imaging, has additional properties such as examination of vascularity degree of tissue. This information can be showed by first-pass perfusion technique and the late-enhancement MR imaging study. The purpose of this paper is to describe MR Imaging findings of this extremely un frequent presentation of multiple primary cardiac liomyosarcoma in a young boy.
\end{abstract}

Volume 3 Issue 4 - 2015

\author{
Alejandro de la Vega, Marcelo Jeich, Germán \\ Girela, Fernando Barbosa, Mario Grasso, \\ Sebastian Pérez Ascani,Alejandro Schröeder \\ Departamento de Cardiolog,Argentina
}

Correspondence: Alejandro de la Vega, Departamento de Cirugía Cardiovascular, Clínica de Imágenes, Neuquén, Servicio de Diagnóstico por Imágenes, Fundación Médica de Río Negro y Neuquén,Argentina,Email alexdevex@hotmail.com

Received: July 12, 2015 | Published: September 22, 2015

Keywords: Liomyosarcoma

\section{Introduction}

Leiomyosarcoma is a malignant tumor arising from smooth muscle and constitutes only $1 \%$ of all the primary cardiac tumors. It has a predilection for the left cavities, and the most common manifestation is dyspnea and cardiac failure from mitral obstructive symptoms. They can be multiple in $30 \%$ of the cases. MR imaging descriptions are infrequent, but they appear to be isointense or hypo intenseto myocardium on T1-weighted images, hyper intense on T2-weighted images, markedly enhanced with gadolinium contrast material. ${ }^{1,2}$

\section{Case report}

We present a case of a 17-year-old boy hospitalized for an acute episode of extertional dyspnea. The young man referred several episodes of asthenia, fatigue and dizziness effort, for 3 months. The Clinical examination revealed that he also presented progressive weight loss with no related causes which was not associated with decreased intake. Blood tests and electrocardiogram showed that he has no cardiovascular background, and there is no familiar record of a heart disease event. To the physical examination systolic murmur is heard, in aortic focus $3 / 6$ and systolic murmur $3 / 6$ in mitral focus. Chest $\mathrm{x}$ ray was normal. An Echocardiogram is performed to rule out for structural heart disease, which evidences intracavitary homogenously echodense left ventricular mass of 4 centimeter of size, mobile, that involves the anterior papillary muscle and obstruction of left ventricular out tract flow. These findings were confirmed by transesophageal echocardiogram and demonstrated sessil attached of lateral wall of myocardium and obstruction of left ventricular in tract flow too. The Doppler examination doesn't reveal interior flow of the mass.

Without evidence of clear difference between pseudo-tumor mass, from primary or secondary cardiac tumor, the oncology consultation suggested head, neck and body Contrast enhance CT and testicular echography to investigate possible primary extra cardiac tumor, with negative results. The patient was than referred to Cardiac RM for further evaluation.

\section{Diagnosis}

\section{Sequence details}

Images were acquired on 1.5 Tesla Magnetom AVANTO Siemens using the 4 element body Matrix in combination with integrated Iso Center Matrix Coil. MR Imaging is used to assess malignant cardiac mass with multiparametric protocol. This protocol consists of anatomic images in sagittal, coronal and axial orientation (e.g. fast low-angle shot gradient echo, FLASH), cine-imaging for function (steady-state free precession, True FISP), tissue characterization with various T1weighted and T2-weighted spin-echo sequences with and without fat suppression, and finally imaging for vascularization assessment with dynamic perfusion evaluation using First-pass perfusion sequence over the mass. Late gadolinium enhancement imaging using PSIR (Phase Sensitive Invertion Recovery) Sequence at dose $0.1 \mathrm{mmmol} /$ $\mathrm{kg}$.

All images are acquired in classical cardiac plans and specific plans over the mass (Figure 1-3).

\section{Imaging findings}

The cardiac MR Imaging showed a first dominant intracavitary left ventricular solid mass of large size measuring 6 by $5 \mathrm{~cm}$ in diameter, irregular with ill-defined borders, with sessile morphology. This Isointense in T1-wieghted SE sequence and Cine True FISP; and hyper intense mass in T2-weighted spin-echo sequences with and without fat suppression with subtle heterogeneous signal is adherent to the antero-lateral wall of myocardium in medial segment (Model of 17 segments of left ventricle by AHA) with direct invasion to the myocardium and the adjacent anterior and posterior papillary muscle confirmed on late enhancement images.

The dynamic perfusion sequence in four-chambers view and short axis and left ventricle long axis view showed global hypo perfusion mass with small area of high perfusion over the anterior papillary muscle involved by the tumor. 
The $\mathrm{T} 1$ post contrast acquired early images showed homogeneous enhance of gadolinium. The late enhancement study shows a heterogeneous pattern of uptake of contrast, with hyper signal over adherent myocardium with direct invasion. The True FISP cine images demonstrated normal left ventricular systolic function and confirmed left ventricle in-flow and out-flow tract obstruction with mass prolapse over the mitral valve with involvement of anterior mitral valve leaflet, with mitral regurgitation.

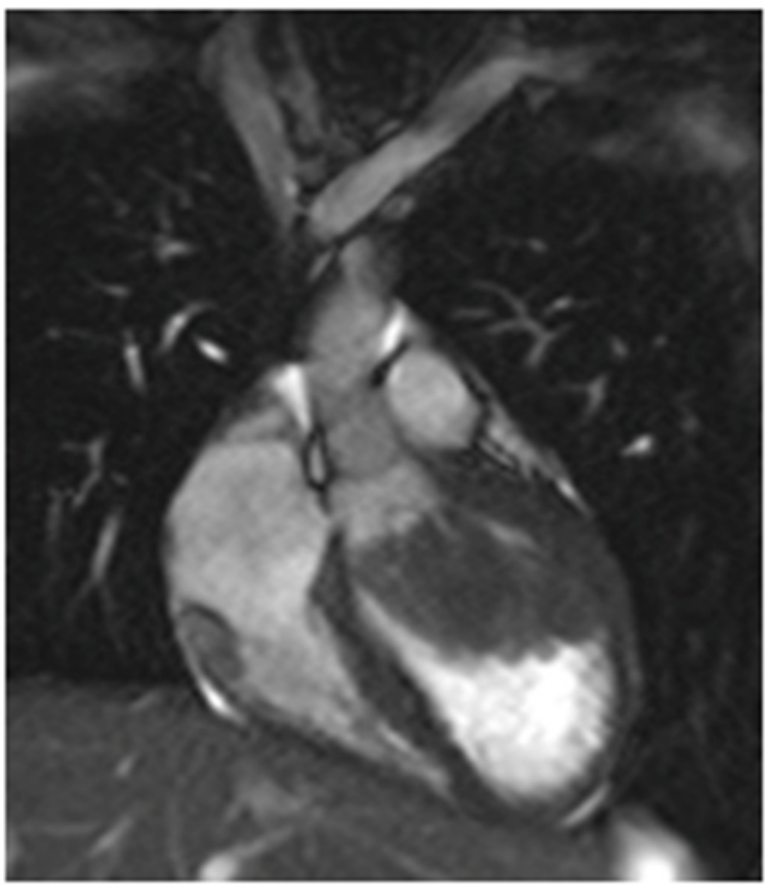

Figure I Left Ventricular out tract flow of cine True FISP SSFP sequence showed intracavitary left ventricular mass sessil, mobile, with obstructive effect over left ventricular out tract blood flow (long arrow); and right ventricular intramiocardial small mass adjacent to atrioventricular sulcus with involvement of tricuspid annulus (short arrow).

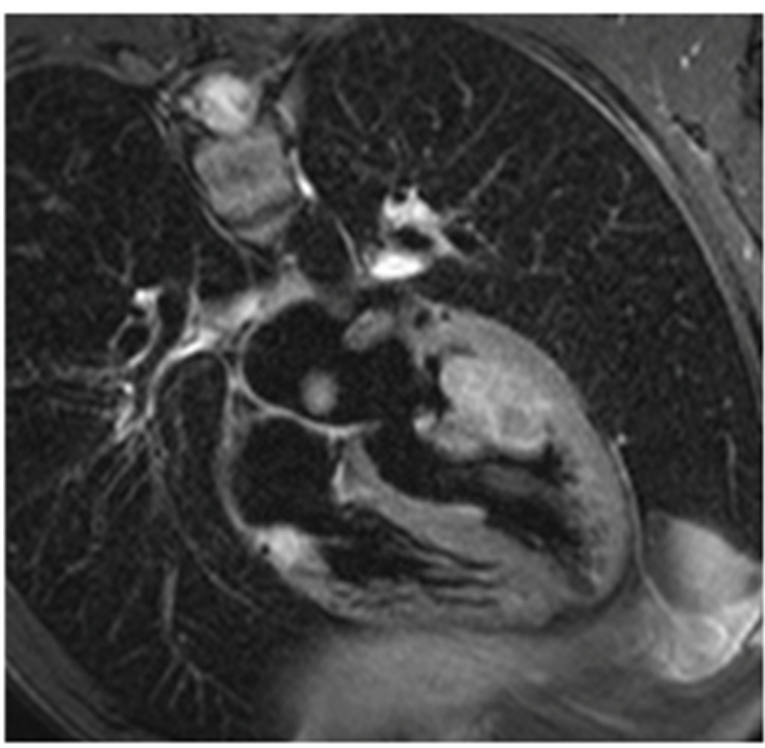

Figure 2 Four-chamber view of T2-weighted spin-echo sequences with fat suppression intracavitary solid hyperintense mass with ill-defined borders, with sessile morphology attached to anterolatero-basal wall (long arrow) and right ventricular intramiocardial hyperintense small mass adjacent to atrioventricular sulcus with involvement of tricuspid annulus (short arrow)
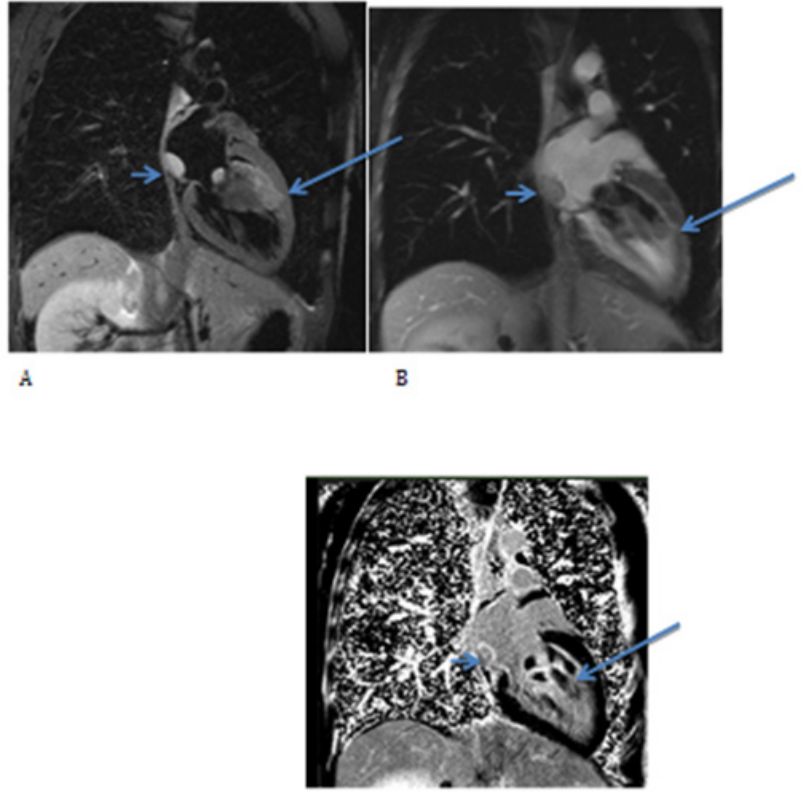

Figure 3 Left ventricle long axis views demonstrate intracavitary left ventricular hyperintense and subtle heterogeneous sessil mass with direct invasion of myocardium at anterior medial segment wall, with obstructive effect over left ventricular in tract blood flow (long arrow) on T2 SE Fat Supression A); and heterogeneous enhancement on TI SE earlier post Gadolinium B) and late enhancement PSIR sequence C).A intracavitary tumor in postero-inferior wall of the left atrium (short arrow), sessil with smooth margins, hyperintense at T2 fat suppression images A) and homogeneousenhancement on TI SE earlier post Gadolinium B), and late enhancement PSIR sequence C).

A second intra cavitary tumor is detected in the postero-inferior wall of the left atrium, small regular and sessil mass with smooth margins, with similar signal in T1, T2 and T2 fat suppression images, Hypo perfused and homogeneous uptakes of gadolinium in early and late enhancement sequence images. Cine TrueFISP sequence reveals normal motility of the left atrial wall.

A third right ventricular intra myocardial infiltrating tumor is detected with transmural involvement of the atrium ventricular sulcus and the tricuspid annulus without compromise of the medial segment of the right coronary artery. This tumor is identified by hyper signal in $\mathrm{T} 2$ and $\mathrm{T} 2$ fat suppression images, irregular with ill-defined borders.

\section{Resolution}

A surgical procedure was decided due to the extensive involvement of intracardiac structures by the different masses and progressive symptoms of left ventricular obstruction. Surgical resection of the larger tumors located in the left atrium and left ventricle was decided. The practice included a replacement of the mitral valve. During the surgical approach, another similar small size tumor is visualized on the anterior face of left ventricle parallel to the upstream anterior descending coronary artery. The patient evolves with a cardiogenic shock after surgical procedure. The transesophageal echocardiogram shows a worsened severe biventricular systolic function. So, an aortic counter pulsation balloon with inotropic support is indicated. Six hours after the surgery the patient evolves with fatal arrhythmia. The pathological study of the pieces consisted of firm tissue with color varying from white to grey. Based on histological and immuno histochemical stainings, it was concluded that the tumors were Leiomyosarcoma. 


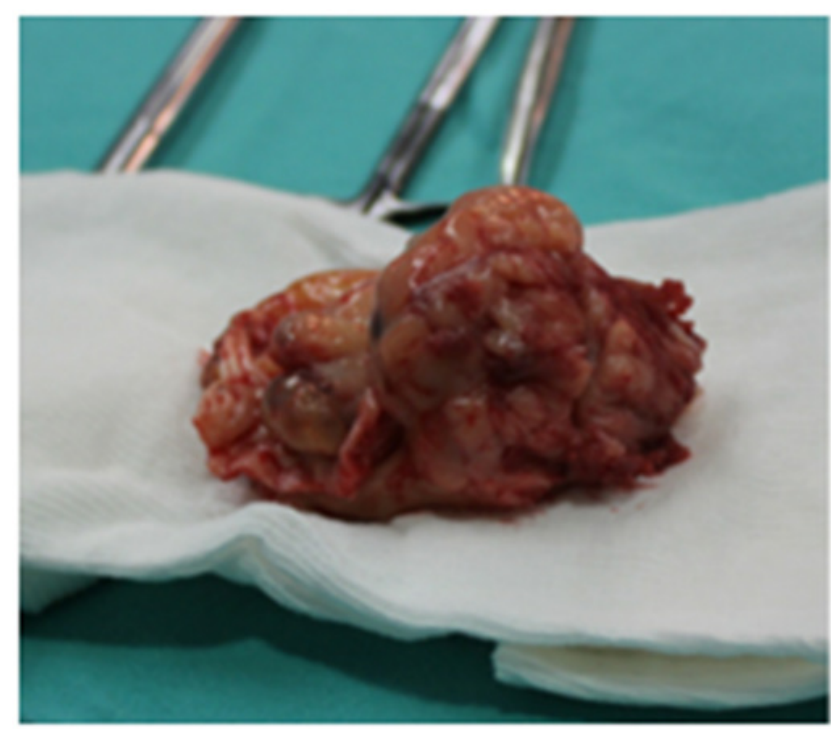

Figure 4 Piece of intracavitary left ventricle.

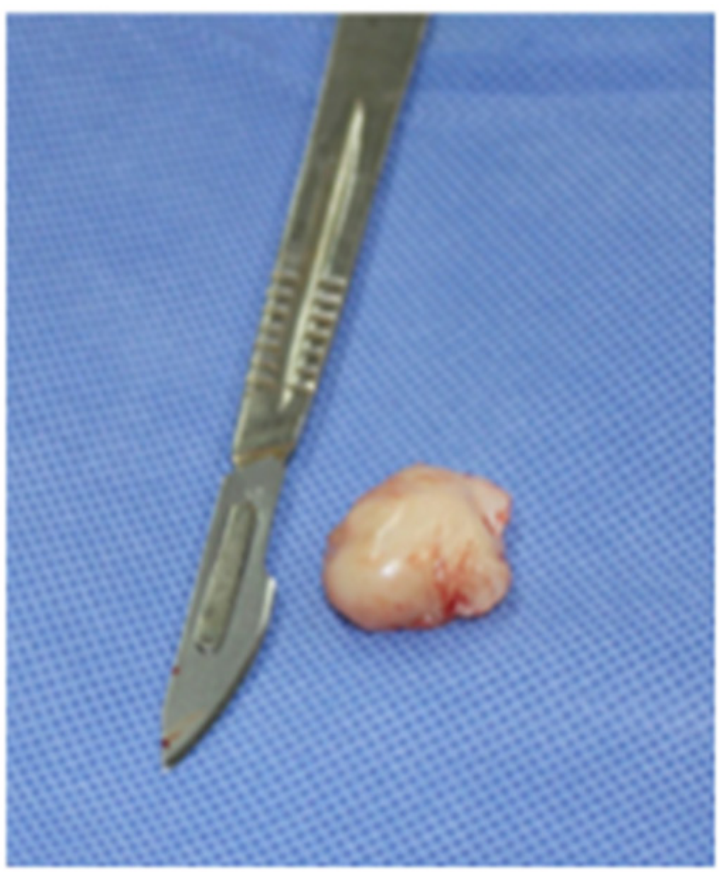

Figure 5 Piece of intracavitary left atrium.

\section{Discussion}

Primary cardiac malignancies (PCM) are a rare condition, actually there are a few cases reported. ${ }^{3}$ From the few hundred cases of malignant heart tumors reported, most have been based on autopsy series. The prevalence of primary cardiac malignancies has been estimated at only $0.001 \%-0.28 \%{ }^{4}$ Inside this group Leiomyosarcoma is the less frequent cardiac tumor representing $0.0019 \%$. The leiomyosarcoma arises from smooth muscle cells and is highly aggressive. ${ }^{5}$

Among malignant primary cardiac tumors, the most reported are those histo pathologically considered as undifferentiated, followed by angiosarcomas. ${ }^{6}$ Being the leiomyosarcomas the rarest of all cases. ${ }^{4}$ Due to delayed presentation of patients at examination there is frequently a systemic spread at the time of diagnosis. As a result management of this condition is difficult and controversial. ${ }^{7}$ Among the malignant primary tumors this case affected patients typically between the third and 4th decade of life which is slightly younger than the average age at presentation for other cardiac pathologies. ${ }^{8}$ Patients usually experience symptoms when the tumor has already advanced severely and are secondary to obliteration of the cardiac chambers and major vessels ${ }^{8}$ (Figure 4 \& 5).

Transthoracic echocardiography is the most readily available noninvasive imaging technique and thus remains the first-line diagnostic test when a cardiac tumor is suspected. Echocardiography demonstrates characteristic anatomic location, morphology, and functional impact. ${ }^{9}$ Transesophageal echocardiography can offer additional imaging planes for further assessment of a lesion, but this is an invasive test. ${ }^{10}$ Magnetic resonance (MR) imaging features of common cardiac masses is therefore important for establishing an accurate diagnosis and for guiding staging, prognosis, and appropriate therapy in confirmed tumors. ${ }^{10}$ Cardiac MR imaging provides advanced tissue characterization and have been shown to accurately differentiate between benign and malignant tumors, and differentiate malignancies within them. ${ }^{11-13}$

Although various combinations of treatments including surgery and resection, tumor surveillance program and postoperatively tumor dissemination control, have been advocated in leiomyosarcomas the prognosis has a very low rate. The survival rate is approximately unanimous throughout the cases studied and communicated, about 12 months of life. ${ }^{14}$ Concerning chemotherapy, the efficacy is still unknown. ${ }^{15,16}$ The optimum treatment of Leiomyosarcoma is not known.

\section{Conclusion}

From the several reports in literature, patients subjected to radical surgery, treatment including heart transplantation, complete removal of the tumor and monitored with drug therapies have 24 months estimated survival. ${ }^{16}$ In our patient, symptoms progress in left ventricular obstruction raises the question of whether surgery should be accelerated or not. The therapeutic dilemma in our patient is the vital potential risk involved in myocardial tumor resection with direct invasion and contractile result in functional impairment that can result from this surgical maneuver.

\section{Acknowledgments}

None.

\section{Conflicts of interest}

None.

\section{Funding}

None.

\section{References}

1. Durand E, Vanel D, Mousseaux E, et al. A recurrent left atrium leiomyosarcoma. Eur Radiol. 1998; 8(1):97-99.

2. Clarke NR, Mohiaddin RH, Westaby S, et al. Multifocal cardiac leiomyosarcoma: diagnosis and surveillance by transoesophageal echocardiog- raphy and contrast enhanced cardiovascular mag- netic resonance. Postgrad Med J. 2002;78(922):492-493.

3. Reynen K. Frequency of primary tumors of the heart. Am J Cardiol. 1996;77(1):107 
4. Rikke E Andersen, Bjarne W Kristensen, Sabine Gill. Cardiac leiomyosarcoma, a case report. Int $J$ Clin Exp Pathol. 2013;6(6):1197-1199.

5. Lam KY, Dickens P, Chan AC. Tumors of the heart: a 20-year experience with a review of 12,485 consecutive autopsies [in German]. Arch Pathol Lab Med. 1993;117(10):1027-1031.

6. Donsbeck AV, Ranchere D, Coindre JM, et al. Primary cardiac sarcomas: an immunohistochem- ical and grading study with long-term fol- low-up of 24 cases. Histopathology. 1999;34(4):295-304.

7. Hamidi M, Moody JS, Weigel TL, et al. Primary cardiac sarcoma. Ann Thorac Surg. 2010;90(1):176-181

8. Burke AP, Cowan D, Virmani R. Primary sarcomas of the heart. Cancer 1992;69(2):387-395

9. Altbach MI, Squire SW, Kudithipudi V, et al. Cardiac MRI is complementary to echocardiography in the assessment of cardiac masses. Echo- cardiography. 2007;24(3):286-300.

10. Sparrow PJ, Kurian JB, Jones TR, et al. MR imaging of cardiac tumors Radio Graphics. 2005;25(5):1255-1276.
11. Kiaffas MG, Powell AJ, Geva T. Magnetic resonance imaging evaluation of cardiac tumor characteristics in infants and chil- dren. Am J Cardiol. 2002;89(10):1229-1233

12. Fieno DS, Saouaf R, Thomson LE, et al. Cardiovas- cular magnetic resonance of primary tumors of the heart: a review. $J$ CardiovascMagnReson. 2006;8(6):839-853.

13. Restrepo CS, Largoza A, Lemos DF, et al. CT and MR imaging findings of malignant cardiac tumors. Curr Probl Diagn Radiol. 2005;34(1):111.

14. Elbardissi AW, Dearani JA, Daly RC, et al. Survival after resection of primary cardiac tumors: a 48-year experience. Circulation. 2008;118(14 Suppl):S7-S15.

15. Malyshev M, Safuanov A, Gladyshev I, et al. Primary left atrial leiomyosarcoma: Literature review and lessons of a case. Asian Cardiovasc Thorac Ann. 2006;14(5):435-440.

16. Hattori $\mathrm{Y}$, Iriyama $\mathrm{T}$, Watanabe $\mathrm{K}$, et al. Rapidly growing primary cardiac leiomyosarcoma. Surg Today. 2000;30(9):838-840. 